\title{
High Rate of Detection of Human ESPL1-HBV S Fusion Gene in Patients With HBV-related Liver Cancer: A Chinese Case-Control Study
}

\author{
BOBIN HU ${ }^{1 *}$, WEN HUANG ${ }^{2 *}$, RONGMING WANG ${ }^{1}$, WEIWEI ZANG ${ }^{1}$, MINGHUA SU $^{1}$, HUIJIAO LI ${ }^{1}$, \\ HUIWEN WANG ${ }^{1}$, BIANCHUAN CAO ${ }^{1}$, DELI DENG ${ }^{1}$, QINGDI QUENTIN LI $^{3}$ and JIANNING JIANG ${ }^{1}$ \\ ${ }^{1}$ Department of Infectious Diseases, The First Affiliated Hospital of Guangxi Medical University, Nanning, P.R. China; \\ ${ }^{2}$ Department of Pathology, First Affiliated Hospital of USTC, Division of Life Sciences and Medicine, \\ University of Science and Technology of China, Hefei, P.R. China; \\ ${ }^{3}$ Center for Cancer Research, National Cancer Institute and Center for Scientific Review, \\ National Institutes of Health, Bethesda, MD, U.S.A.
}

\begin{abstract}
Aim: It has been shown that the integration of hepatitis $B$ virus $(H B V)$ gene into the host genome is a high-risk factor for development of hepatocellular carcinoma (HCC). However, the relationship between HBV S-integrated human extra spindle pole bodies-like 1 (ESPL1) gene and HCC is unknown. This study was designed to detect HBV S-integrated human ESPLI fusion gene in patients with HCC for potentially using this fusion gene as a biomarker for HCC diagnosis. Patients and Methods: Nineteen and 70 patients with chronic hepatitis $B(C H B)$ were recruited to the experimental and control groups, respectively, and both groups underwent an effective nucleoside/nucleotide analog therapy and follow-up for HCC occurrence for up to 11 years. HCC tissues were obtained by surgical resection from the experimental group, while liver tissues were collected by liver biopsy in the control group prior to treatment with nucleoside/nucleotide analogs. Alu polymerase chain reaction was used to assess $H B V S$ gene integration in the liver tissues from both groups. HBV S-integrated human ESPL1 fusion gene was then detected in patients with $H B V S$ gene integration using a gene database. Results: All patients in the
\end{abstract}

This article is freely accessible online.

*These Authors contributed equally to this work.

Correspondence to: Dr. Jianning Jiang, Department of Infectious Diseases, First Affiliated Hospital of Guangxi Medical University, 6 Shuangyong Road, Nanning 530021, P.R. China. E-mail: jjianning@163.com; Dr. Quentin Li, Center for Scientific Review, National Institutes of Health, 6701 Rockledge Drive, Bethesda, MD 20892, U.S.A. E-mail: quentin.li@nih.gov

Key Words: Integration, fusion gene, HBV S, human ESPL1, hepatocellular carcinoma, biomarker. experimental group developed HCC, whereas no HCC was diagnosed in the control group. HBV S gene integration was identified in 12 out of 19 HCC tissues in the experimental group, giving a detection rate of $63.2 \%$, which was significantly greater than that of $15.7 \%(11 / 70)$ in the control group $(p<0.001)$. We further showed that HBV S-integrated human ESPL1 fusion gene was detected in eight patients (rate of 66.7\%) among the 12 patients with HCC with HBV $S$ gene integration in the experimental group, whereas the fusion gene was not detectable in any of the patients in the control group $(p=0.001)$. Conclusion: This research demonstrates a high detection rate of $H B V$ S-integrated human ESPL1 fusion gene in patients with $H B V$-related HCC and shows that this fusion gene appears to be associated with HCC development in patients with CHB. These findings suggest that HBV S-integrated human ESPL1 fusion gene may potentially serve as a biomarker for early detection of $H C C$ in $H B V$-infected populations.

Hepatitis B virus (HBV) infection is a major hygiene and health problem worldwide as well as the main cause of hepatic inflammation, liver cirrhosis, and hepatocellular carcinoma (HCC). It is estimated that there are 2.4 hundred million HBV-infected patients in the world, and 60 million of them may eventually die from HCC, an aggressive form of liver cancer. It has been shown that there is a strong relationship between $\mathrm{HBV}$ infection and $\mathrm{HCC}$ and that intrahepatic $\mathrm{HBV}$ replication is significantly correlated with HCC occurrence (1-3). Therefore, prevention of $\mathrm{HBV}$ infection has been proposed as an effective way of reducing $\mathrm{HBV}$-related HCC incidence, and anti-HBV treatment is given more and more attention in regard to reduction of $\mathrm{HCC}$ occurrence (4-9). Nucleoside/nucleotide analog (NUC) therapy of HBV infection is widely accepted because of its oral convenience, strong antiviral effect, and high safety. 


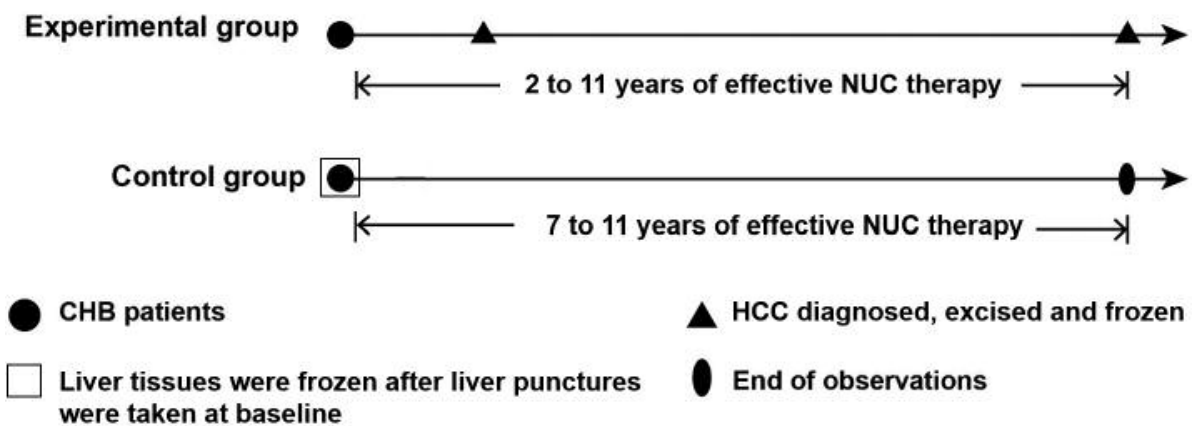

Figure 1. Schematic diagram of the experimental design of this study. Patients with chronic hepatitis B (CHB) in both the experimental group and the control group underwent an effective nucleoside/nucleotide analog (NUC) therapy and follow-up observation for hepatocellular carcinoma (HCC) development for up to 11 years. In the experimental group, HCC was diagnosed in all patients with CHB at a time frame of between 2 and 11 years after receiving NUC therapy, whereas no HCC was observed in the control group during 11 years of treatment and follow-up. HCC tissues were obtained by surgical resection in the experimental group when HCC was diagnosed, while liver tissues were collected by liver biopsy in patients of the control group prior to NUC treatment.

Despite the antiviral effectiveness of NUC treatment in HBV infection, a small proportion of $\mathrm{HBV}$-infected patients still develop HCC after a long-term effective NUC therapy, especially those with occult HBV infection or with lower HBV DNA expression levels which are beyond assay detection sensitivity and efficiency (10).

Clinically, $80 \%$ of patients with HCC have HBV-related HCC (11), suggesting an important role of HBV infection in the development of HCC. Increasing research has confirmed that $\mathrm{HBV}$ infection and $\mathrm{HBV}$ gene integration are involved in the pathogenesis of HCC. Although the mechanism is not completely understood, evidence shows that induction of genetic instability during HBV DNA integration into the host cell chromosomes, generation of insertional mutagenesis in cancer-associated cellular genes, and activation of proto-oncogenes due to HBV gene integration to the specific sites and regions of the nearby host genes may account for the carcinogenesis through HBV gene integration in HCC development (12-17). Currently, most studies have focused on the relationship between HBV $\mathrm{X}$ gene integration and HCC development $(12,18)$. While HBV S gene integration is regarded as a small probability event, there is a lack of extensive and profound investigations exploring the role of HBV S gene integration in the oncogenesis and tumorigenicity of human liver cancer. We recently showed that HBV S gene integration is a crucial factor leading to a sustained high level of hepatitis B surface antigen ( $\mathrm{HBsAg}$ ) in patients with chronic hepatitis B (CHB) following antiviral therapy (19). Accumulating evidence also shows that persistent expression of integrated HBV DNA and sustained higher level of HBsAg are risk factors for HCC occurrence in patients with CHB (20-22). However, NUC treatment of $\mathrm{HBV}$ infection cannot completely block HCC occurrence in the clinic because these drugs do not act on the HBV genes integrated into the genome of the host cell.

The role of HBV S gene integration in the development of HCC is unknown. In the current study, we conducted a case-control clinical study to investigate the relationship between HBV S integration and HCC.

\section{Patients and Methods}

Patient selection. This project was a prospective study. The patients for the study were selected from those with CHB who then underwent NUC therapy and a long-term follow-up cohort. During the follow-up period, all patients with $\mathrm{CHB}$ underwent abdominal ultrasound or computed tomography every 6 months. When a liver mass was found, the patient underwent contrast-enhanced ultrasound or enhanced magnetic resonance imaging examination in order to facilitate early detection of liver tumors. The inclusion criteria of the patients were as follows: for the experimental group, patients with CHB were provided with effective NUC therapy for 2-11 years; during this period of time, when HCC occurred and was confirmed by histopathological diagnosis, the patients underwent surgical excision and HCC tissues were cryopreserved. For the control group, prior to NUC treatment, patients underwent liver puncture biopsy and the liver tissues were conserved. The patients then received 7-11 years of NUC therapy and follow-up as above. The exclusion criteria were as follows: (i) Co-infection with hepatitis $\mathrm{C}$ virus, hepatitis $\mathrm{D}$ virus, or human immunodeficiency virus; (ii) a history of interferon therapy; (iii) diagnosis of HCC at baseline; and (iv) lost during the follow-up period. After excluding 256 patients who did not meet the inclusion criteria, one patient who not have surgical excision, and 93 patients who were lost during the follow-up time, 19 patients were eligible and enrolled in the experimental group and 70 patients were selected for the control group (Figure 1). The use of human specimens from patients in the present study was approved by the Ethics Committee of the First Affiliated Hospital of Guangxi Medical University (approval number: 2019-K-Y-E-193), and all experiments were performed in accordance with the ethical standards of the 1995 Declaration of 
Hu et al: Detection of Human ESPL1-HBV S Fusion Gene

Table I. Primers for Alu-polymerase chain reaction analysis of hepatitis virus B S gene integration used in this study.

\begin{tabular}{|c|c|c|c|}
\hline Primer* & Sequence $\left(5^{\prime} \rightarrow 3^{\prime}\right)$ & Location & \\
\hline UP5 & $\begin{array}{l}\text { CAGUGCCAAGUGUUUGCUGACG } \\
\text { CCAAAGUGCUGGGGAUUA }\end{array}$ & & Forward sequence \\
\hline T3-515 & $\begin{array}{l}\text { AUUAACCCUCACUAAAGCCUCGAU } \\
\text { AGAUYRYRCCAYUGCAC }\end{array}$ & & Reverse sequence \\
\hline $\begin{array}{l}\text { UP6 } \\
\text { Mid-T3 }\end{array}$ & $\begin{array}{l}\text { CAAGTGTTTGCTGACGCCAAAG } \\
\text { ATTAACCCTCACTAAAGCCTCG }\end{array}$ & & Forward sequence (Tag) \\
\hline 52821UF & CACCAUAUUCUUGGGAACAAG & 2818-2838 & $\begin{array}{l}\text { Reverse sequence (Tag) } \\
\text { HBV S1 }\end{array}$ \\
\hline $\begin{array}{l}3031 \mathrm{~F} \\
3132 \mathrm{~F}\end{array}$ & $\begin{array}{l}\text { GGAGCATTCGGGCCAGGGTTC } \\
\text { TGCCTCCACCAATCGGCAGT }\end{array}$ & $\begin{array}{c}3028-2048 \\
282-301\end{array}$ & $\begin{array}{l}\text { HBV S2 } \\
\text { HBV S3 }\end{array}$ \\
\hline
\end{tabular}

*Primers design reference PubMed Nucleotide GenBank: V00866. The tag sequences of the primers UP5 and T3-515 are underlined.

Helsinki, as revised in Tokyo in 2004. Written informed consent was obtained from all patients.

Sample collection and storage. HCC tissue specimens were collected by surgical resection in the experimental group $(5 \mathrm{mg})$, and liver puncture biopsy specimens were collected from the control group prior to NUC therapy $(5 \mathrm{mg})$. All the samples from both groups were kept frozen at $-80^{\circ} \mathrm{C}$.

Histopathology and immunohistochemistry. For histopathological examination, paraffin-embedded sections were stained with hematoxylin and eosin (HE) and were sealed with neutral gum.

Immunohistochemical assay was performed using a Streptomyces antibiotic protein-peroxidase ligation method. In brief, 5 - $\mu \mathrm{m}$ tissue sections were cut from formalin-fixed, paraffin-embedded specimens. The expression of HBsAg protein was measured with UltraSensitive ${ }^{\mathrm{TM}}$ S-P Kit (Maixin, Fuzhou, Fujian, PR China). Before labeling with primary antibody, the sections were deparaffinized, antigens were restored, and the sections were incubated with hydrogen peroxide to block endogenous peroxidase. The specimen sections were incubated overnight at $4^{\circ} \mathrm{C}$ with primary antibody to $\mathrm{HBsAg}$ (1:2000; Maixin) and then with a secondary antibody (1:2000; Maixin) conjugated to streptavidin-peroxidase. After color development with diaminobenzidine peroxidase substrate solution, the sections were counterstained with hematoxylin. For the negative control, phosphatebuffered saline was used instead of primary antibody. The immunohistochemical results were examined independently by two pathologists. When all of the cytoplasm exhibited a brown reaction product, sections were considered positive. The sections were examined under a microscope at low power to identify evenly labeled areas. Images were acquired using a Leica DMRD optical microscope (Leica, Wetzlar, Germany) at 100-200× magnification.

Detection of $H B V S$ gene integration. DNA was isolated from the excisional hepatocellular carcinoma tissues and liver biopsy specimens using DNeasy Tissue Kit (Qiagen Bio-engineering Co., Ltd., Dusseldorf, Germany). The Alu polymerase chain reaction (PCR) assay was used to verify HBV S gene integration into the hepatocyte genome (see Table I for the primers used in this study). The amplified products were electrophoresed on $1 \%$ agarose gel, recycled, and checked by sequencing (Guangzhou Biological Engineering Co., Ltd., Guangzhou, PR China).
Bioinformatic and statistical analysis. Statistical analyses for comparisons among groups were performed with SPSS software version 22.0 (IBM, Armonk, NY, USA). The continuous variables are represented by the mean \pm standard deviation (SD). Categorical variables are summarized as counts and percentage. The rates of HBV S gene integration were compared between the experimental group and the control group by chi-square test of a four-fold table. The detection rate of human ESPL1-HBV S fusion gene of the patients with HBV S gene integration was compared between the experimental and the control groups by Fisher's exact probability. The expression of human ESPL1 gene in normal liver tissues and liver cancer tissues was searched from the GeneCloud Biotechnology Information (GCBI) gene database, and then compared by independent samples $t$-test. Statistical significance was defined as a $p$-value of less than 0.05. All statistical tests were two-sided. The human ESPL1-HBV S fusion gene was analyzed by using the NCBI, Ensembl, and GeneCards gene databases.

\section{Results}

Patients. All the patients entered in the study were Chinese. Nineteen patients in the experimental group underwent 2 to 11 years of effective NUC therapy and HCC developed in these patients at an average of 4.8 years. Seventy patients in the control group underwent 7 to 11 years of effective NUC therapy and had no HCC during an average of 7.8 years. The general information of the patients in the experimental and the control groups is given in Table II.

Histopathological and immunohistochemical diagnosis of $H B V$ infection and HCC. The liver tissue samples from surgical resection in the experimental group were confirmed to be HCC by HE staining (Figure 2A). In the control group, chronic inflammation and fibrosis degree in the liver biopsy tissues from patients with $\mathrm{CHB}$ was shown to be at least G2S2 (Figure 2B). Immunohistochemical staining of the liver tissues from the control group showed HBsAg positivity (Figure 2C). 
Rate of $H B V S$ gene integration is higher in patients with $H C C$ than in those with $\mathrm{CHB}$ alone. The detection rate of HBV S gene integration was compared by chi-square test of four-fold table. As can be seen in Table II, 63.2\% (12/19) patients in the experimental group had HBV $\mathrm{S}$ gene integration in the HCC tissues, while only $15.7 \%(11 / 70)$ patients in the control group had HBV S gene integration in the liver puncture biopsy tissues at baseline prior to NUC treatment. The integration rate of $\mathrm{HBV} S$ gene in the experimental group was significantly higher than that in the control group $\left(\chi^{2}=17.5, p<0.001\right)$.

Human ESPL1-HBV $S$ fusion gene is detected in HCC patients but not in $C H B$ patients. We used gene sequence analysis software to identify the fusion gene at the sites of HBV S gene integration, and applied Fisher's exact probability to compare the detection rate of human ESPL1HBV S fusion gene between the experimental group and the control group. The detection rate of human ESPL1-HBV S fusion gene was $66.7 \%(8 / 12)$ in the experimental group, but 0 in the control group, which was a statistically significant difference $\left(\chi^{2}=10.7, p=0.001\right)$.

Bioinformatic analysis of human ESPL1-HBV S fusion gene. We searched NCBI, Ensembl, and GeneCards gene databases for ESPL1 gene We then analyzed the sequence of the human ESPL1-HBV S fusion gene in eight patients with HCC in the experimental group by gene database and identified a consensus sequence of CTGGGAACCCCCGAATG in both the human ESPLI gene and HBV S gene. This sequence is a part of the human ESPLI coding sequence and is connected to the HBV S gene SP II promoter, as illustrated in Figure 3.

Differential expression of human ESPL1 gene between HCC tissues and normal tissues. We searched and compared the expressive abundance data of human ESPL1 gene between different normal tissues and different tumor tissues from the GCBI database and found that the expression of human ESPL1 gene was significantly higher in most of the tumor tissues assessed, including HCC tissues, than in normal tissues (301.2 vs. 104, $p<0.01)$, as shown in Figure 4.

Human ESPL1-HBV S fusion gene in patients with CHB is associated with HCC development. To understand the role of human ESPL1-HBV S fusion gene in HCC development, we carried out a clinical study in the experimental and control groups. Both HBV S gene integration and human ESPL1HBV S fusion gene were detected in eight patients with CHB in the experimental group, whereas only HBV $\mathrm{S}$ gene integration, but not human ESPLI-HBV S fusion gene was detected in 11 patients with CHB in the control group. All patients in both groups received an effective NUC therapy
Table II. Selected patient characteristics. Continuous variables are expressed as mean \pm standard deviation $(S D)$.

\begin{tabular}{lcc}
\hline Factor & $\begin{array}{c}\text { Experimental } \\
\text { group }\end{array}$ & $\begin{array}{c}\text { Control } \\
\text { group }\end{array}$ \\
\hline Total number of patients & 19 & 70 \\
Age, years & $48.7 \pm 11.3$ & $45.5 \pm 9.2$ \\
Male, n (\%) & $17(89.5)$ & $50(71.4)$ \\
Effective therapy time, years & $4.8 \pm 1.6$ & $7.8 \pm 1.1$ \\
History positive, $\mathrm{n}(\%)$ & $16(84.2)$ & $39(55.7)$ \\
Rate of S gene integration, $\mathrm{n}(\%)$ & $12(63.2)$ & $11(15.7)$ \\
Detection rate of fusion gene, $\mathrm{n}(\%)$ & $8(66.7)$ & $0(0)$ \\
\hline
\end{tabular}

and follow-up in the study. Interestingly, all patients in the experimental group developed HCC starting from 2 to 11 years after effective NUC treatment. In contrast, none of the patients in the control group developed HCC following 7-11 years of effective NUC therapy. These results indicate that human ESPL1-HBV S fusion gene is strongly associated with $\mathrm{HCC}$ in $\mathrm{HBV}$-infected patients.

\section{Discussion}

It is well known that HBV $\mathrm{X}$ gene integrates into the host genome and fuses with some human genes at certain sites and forms human-HBV $\mathrm{X}$ fusion genes leading to $\mathrm{HCC}$ development $(13,23)$. However, little is known about the role of HBV S gene integration and fusion of human and HBV S genes in the pathogenesis of HCC. We carried out a casecontrol study to investigate the relationship between HBV S gene integration and $\mathrm{HCC}$, and we found for the first time a high rate of the human ESPL1-HBV S fusion in patients with HBV-related HCC but not in those with $\mathrm{CHB}$ alone. We further showed that the human ESPL1-HBV S fusion gene is significantly associated with HCC occurrence in patients with CHB following long-term effective NUC therapy.

Human ESPL1 gene, also known as SEPA gene, is a protein coding gene for separase protein, whose main function is to catalyze fibronectin decomposition at mitosis anaphase in order to separate sister chromatids during cell division (24). Studies have reported that mutation of human ESPL1 gene and fusion of the human ESPL1 gene with itself or with viral genes, as well as the expression products of the fusion genes are highly relevant to the development of different tumor types $(12,16,25-27)$. Consistent with the literature, we showed that the rate of HBV S gene integration and the detection rate of human ESPLI-HBV S fusion gene were significantly higher in patients with HCC than in patients with $\mathrm{CHB}$ alone $(p<0.01)$, suggesting the involvement of HBV S gene integration and human ESPL1HBV $\mathrm{S}$ fusion gene in HCC development. 

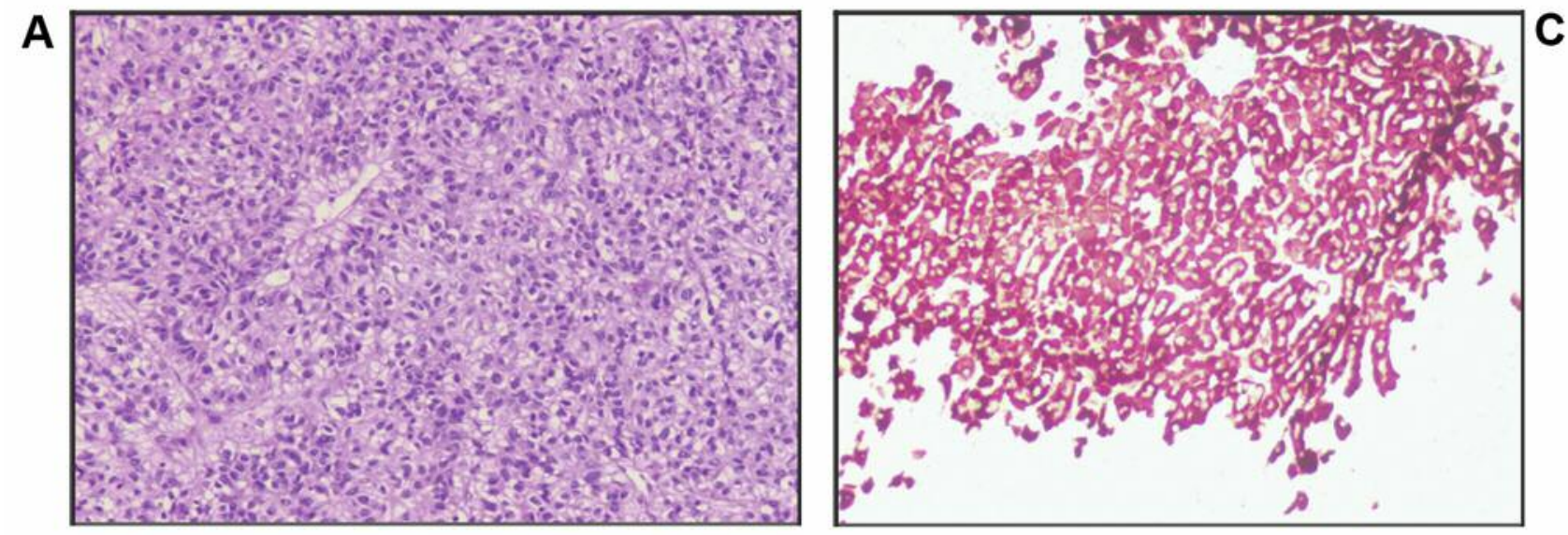

B

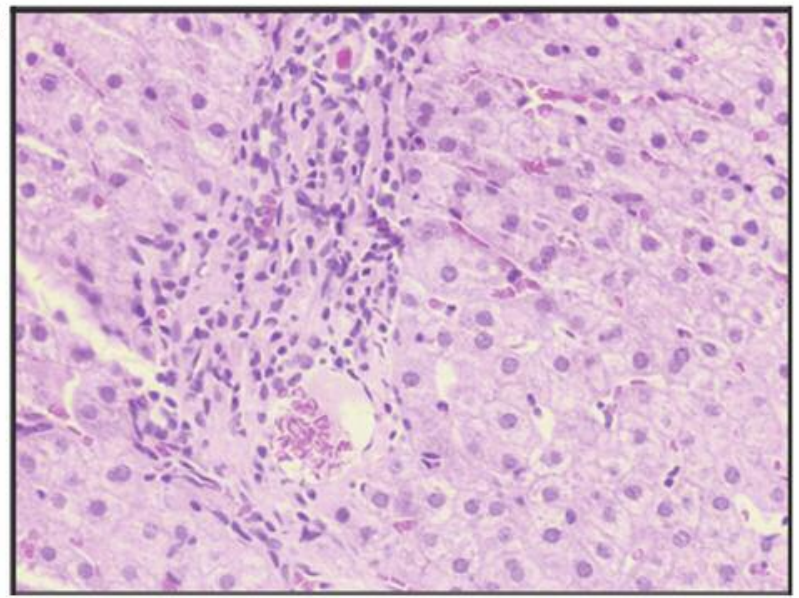

Figure 2. Histopathological and immunohistochemical assessment of the liver tissues from the experimental and control groups. The liver cancer tissues in the experimental group were obtained from tumor resection of patients with hepatocellular carcinoma; the liver tissues in the control group were obtained from liver biopsy of patients with chronic hepatitis $B(C H B)$. Both the HCC $(A)$ and $C H B(B)$ tissues were fixed in $10 \%$ neutral buffered formalin, paraffin embedded, sectioned and stained with hematoxylin and eosin for histopathological examination. Immunohistochemical staining with antibody to HBsAg was positive in cytoplasm in tissues from a patient with $\mathrm{CHB}(\mathrm{C})$. Representative sections of the liver tissues from both groups are shown (original magnification, $\times 200$ ).

A

Human ESPL1 gene in chr12

HBV S gene

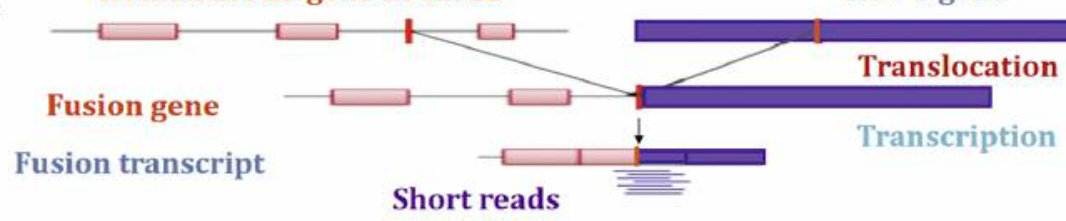

B

WMWMMWMC Human ESPL1-HBVS

chr.12q13.13 coding region GGCTGGGAACCCC̀CGAATG

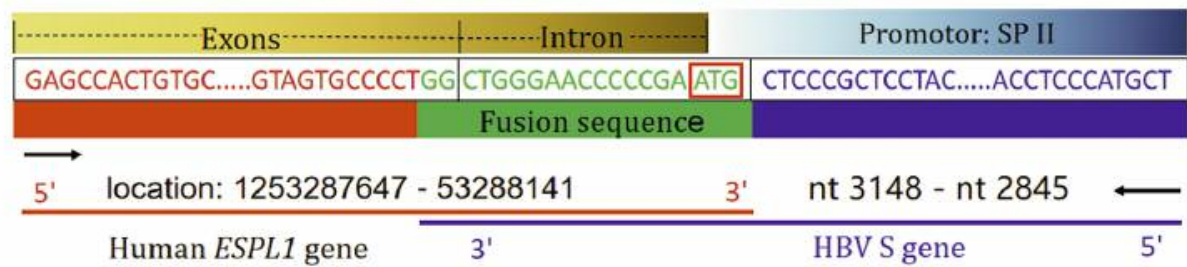

Figure 3. Human extra spindle pole bodies-like 1 (ESPL1)-hepatitis B virus (HBV) S fusion gene. A: The diagram of fusion gene formation by human ESPL1 gene and HBV S gene. B: Analysis of the human ESPL1-HBV S fusion gene sequence. The genomic location of the fusion fragment of human ESPL1 gene is at 1253287647-53288141, which is the coding sequence, including relevant exons and introns. HBV $S$ gene fragment (nt3148-nt2845) is chimeric reservedly into (3'-5') human ESPL1 gene and fuses with it. Furthermore, this sequence is a part of the promoter SP II of the HBV S gene, which is connected to the nearby initiator codon ATG and controls human ESPL1-HBV S fusion gene expression. 
A

Cancer tissues
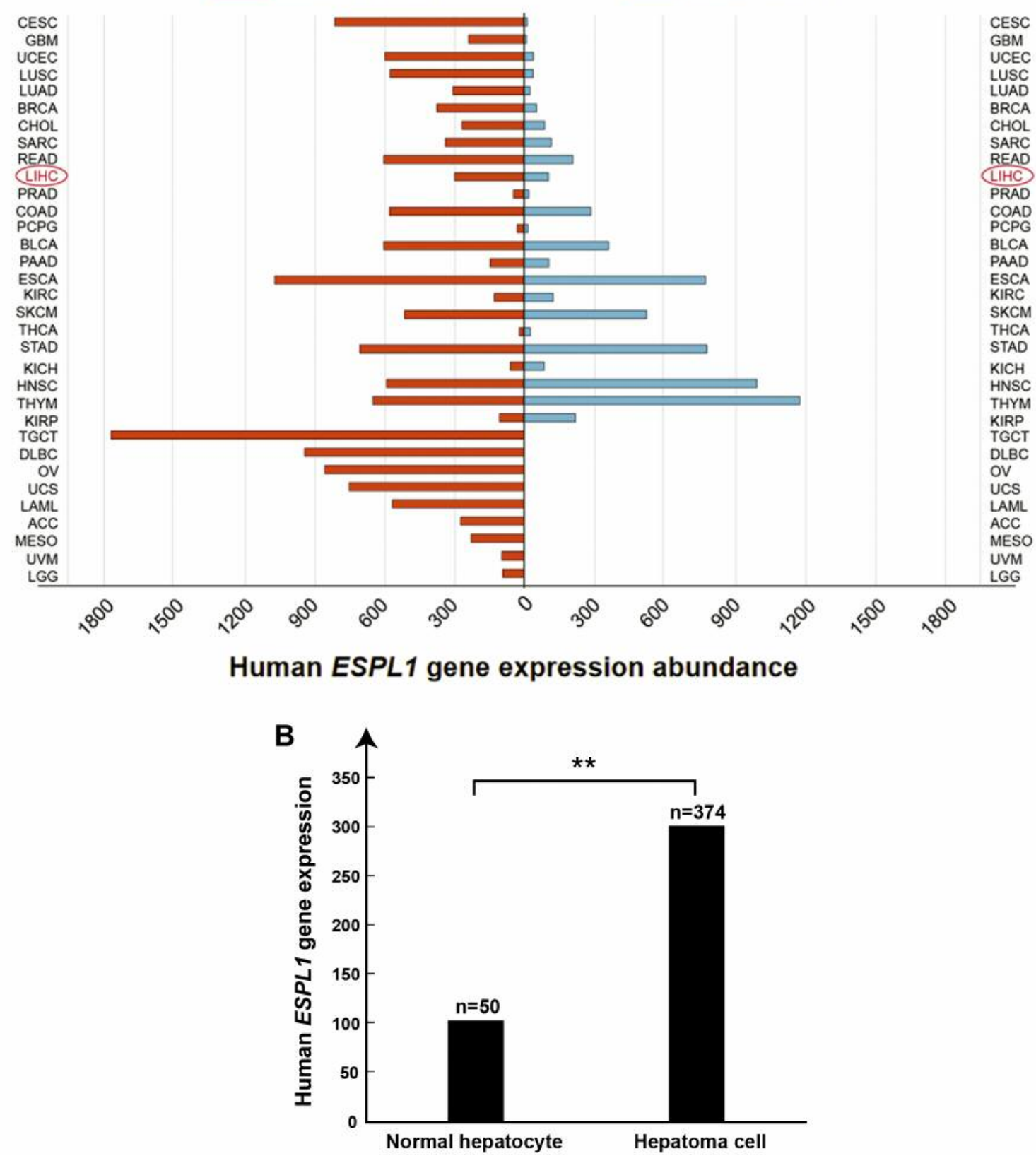

Figure 4. Abundance of expression of human extra spindle pole bodies-like 1 (ESPL1) gene in different tumor and normal tissues. A: Comparison of the abundance of human ESPL1 gene expression in various tumor tissues and normal tissues. Student's t-test was used to analyze the statistical significance of differences between different cancer tissues and different normal tissues. As seen, the level of human ESPL1 gene expression was higher in the liver hepatocellular carcinoma $(L I H C)$ tissue than in the normal liver tissue $(p<0.01)$. B: Comparing human ESPL1 gene expression between normal hepatocytes and hepatocellular carcinoma cells by independent samples t-test; the level of human ESPL1 gene expression in normal hepatocytes was significantly lower than that in hepatocellular carcinoma cells $(* * p<0.01)$. CESC: Cervical squamous cell carcinoma and endocervical adenocarcinoma; GBM: glioblastoma multiforme; UCEC: uterine corpus endometrial carcinoma; LUSC: lung squamous cell carcinoma; LUAD: lung adenocarcinoma; BRCA: breast invasive carcinoma; CHOL: cholangiocarcinoma; SARC: sarcoma; READ: rectum adenocarcinoma; LIHC: liver hepatocellular carcinoma; PRAD: prostate adenocarcinoma; COAD: colon adenocarcinoma; PCPG: pheochromocytoma and paraganglioma; BLCA: bladder urothelial carcinoma; PAAD: pancreatic adenocarcinoma; ESCA: esophageal carcinoma; KIRC: renal clear cell carcinoma; SKCM: skin cutaneous melanoma; THCA: thyroid carcinoma; STAD: stomach adenocarcinoma; KICH: kidney chromophobe; HNSC: head and neck squamous cell carcinoma; THYM: thymoma; KIRP: papillary cell renal carcinoma; TGCT: testicular germ cell tumor; DLBC: diffuse large B-cell lymphoma; OV: ovarian serous cystadenocarcinoma; UCS: uterine carcinosarcoma; LAML: acute myeloid leukemia; ACC: adrenocortical carcinoma; MESO: mesothelioma; UVM: uveal melanoma; LGG: low-grade glioma. 
To further understand the role of HBV infection and human ESPL1-HBV $\mathrm{S}$ fusion gene in this process, we analyzed the sequence and structure of the human ESPL1HBV $S$ fusion gene in eight patients with $\mathrm{HCC}$ in our experimental group by gene database. As illustrated in Figure 3, the human ESPL1 sequence fragment at the fusion site is the coding sequence, including exons and introns. HBV S gene (the base sequence from 3' to 5') backward inserts into the human ESPLI gene sequence and shares the CTGGGAACCCCCGAATG sequence of with human ESPL1 gene. HBV S gene sequence at the fusion site is a part of SP II promoter of HBV virus. Therefore, the expression of the human ESPL1-HBV S fusion gene is under the control of the initiation codon ATG and SP II promoter, which lead to the expression of human ESPL1 gene. This may account for our finding (Figure 4) that the levels of human ESPL1 gene expression were higher in HCC cells and in different tumor tissues as compared with the normal tissues $(28,29)$.

In addition to the increased expression of the gene, the structure and function of the mutated human ESPL1 gene product may also be changed. As a result, altered human ESPL1 protein may cause an error of sister chromatid segregation, production of heteroploidy, instability of the gene, uncontrollability of apoptosis $(30,31)$, and finally tumor occurrence. These mechanisms may underly our study results in which only those patients with $\mathrm{CHB}$ with HBV $\mathrm{S}$ gene integration and detection of human ESPL1-HBV S gene fusion gene developed HCC, even under 2-11 years of effective NUC treatment, whereas patients with $\mathrm{CHB}$ without fusion gene did not develop HCC. Moreover, the patients with $\mathrm{CHB}$ and HBV $S$ gene integration alone in our control group had no HCC occurrence during an up to 11-year period of effective NUC therapy and follow-up, indicating that both HBV $\mathrm{S}$ gene integration and human ESPL1-HBV $\mathrm{S}$ fusion gene are required in the process of $\mathrm{HCC}$ development in $\mathrm{HBV}$-infected patients. It is possible that HBV S gene integration and human ESPL1-HBV S fusion gene, together with other genes, may act coordinately and collaboratively to cause tumor development and to promote tumor progression of $\mathrm{HCC}$ in patients with $\mathrm{CHB}$. However, the relationship between $\mathrm{HBV}$ $\mathrm{S}$ gene integration and HCC occurrence as well as the mechanism for human ESPL1-HBV S fusion gene in causing HCC development remains to be experimentally determined.

Collectively, these findings indicate that patients with $\mathrm{CHB}$ with human ESPL1-HBV S fusion gene are a high-risk population for HCC development and further suggest that this fusion gene may potentially serve as a biomarker for early detection and diagnosis of HCC in HBV-infected populations.

\section{Funding}

This study was supported by grants from the National Natural Science Foundation (81960115), Natural Science Foundation of
Guangxi Key Laboratory for the Prevention and Control of Viral Hepatitis (no. GXCDCKL201802), Science and Technology Major Project of China (no. 2017ZX10202201), and the Key Laboratory of High-Incidence-Tumor Prevention \& Treatment (Guangxi Medical University), Ministry of Education (GKE2019-04).

\section{Conflicts of Interest}

The Authors declare that they do not have any commercial or associative interests that represent a conflict of interests in connection with this study.

\section{Authors' Contributions}

$\mathrm{BH}$ and $\mathrm{WH}$ conducted the studies, performed the experiments, drafted the article, and are the primary Authors of this article. MS performed the experiments. RW, WZ, HL, HW, BC, and DD collected specimens and analyzed the data. JJ and QQL conceived the project, interpreted data, and revised the article. JJ obtained the funding and designed the research. All Authors met the authorship criteria and read and approved the final article.

\section{Acknowledgements}

The Authors sincerely express their thanks for the financial support of the funds for our research (see under Funding) and gratefully acknowledge our colleagues and many individuals for their help during the study processes. We would also like to thank Brigit Sullivan, NIH Library, for reviewing the article.

\section{References}

1 Ott JJ, Stevens GA, Groeger J and Wiersma ST: Global epidemiology of hepatitis B virus infection: New estimates of agespecific HBsAg seroprevalence and endemicity. Vaccine 30: 22122219, 2012. PMID: 22273662. DOI: 10.1016/j.vaccine.2011.12.116

2 Lamontagne RJ, Bagga S and Bouchard MJ: Hepatitis B virus molecular biology and pathogenesis. Hepatoma Res 2: 163-186, 2016. PMID: 28042609. DOI: 10.20517/2394-5079.2016.05

3 Ringelhan M, Heikenwalder M and Protzer U: Direct effects of hepatitis B virus-encoded proteins and chronic infection in liver cancer development. Dig Dis 31: 138-151, 2013. PMID: 23797136. DOI: $10.1159 / 000347209$

4 DeHalegoua MD and Hann HW: Prevention of hepatocellular carcinoma and its recurrence with anti-hepatitis B viral therapy. Minerva Gastroenterol Dietol 60: 191-200, 2014. PMID: 25000955.

5 Dandri $\mathrm{M}$ and Petersen J: Latest developments in the treatment of hepatitis B. Minerva Gastroenterol Dietol 62: 88-102, 2016. PMID: 26448309. DOI: 10.1177/0009922811398390

6 Galle PR, Forner A, Llovet JM, Mazzaferro V, Piscaglia F, Raoul JL, Schirmacher P and Vilgrain V: European Association for the Study of the Liver: EASL Clinical practice guidelines: Management of hepatocellular carcinoma. J Hepatol 69: 182236, 2018. PMID: 29628281. DOI: 10.1016/j.jhep.2018.03.019

7 Yan S-Y, Fan J-G and Qiao L: Hepatitis B virus (HBV) infection and hepatocellular carcinoma-New insights for an old topic. Curr Cancer Drug Targets 17: 505-511, 2017. PMID: 27677954. DOI: $10.2174 / 1568009616666160926124530$ 
8 Lai C-L and Yuen M-F: Prevention of hepatitis B virus-related hepatocellular carcinoma with antiviral therapy. Hepatol 57: 399408, 2013. PMID: 22806323. DOI: 10.1002/hep.25937

9 Fan Y, Liu W, Lei C, Tan X and Cao G: Anti-HBV treatment delays development and evolution of HCC. Acad J Second Military Med Univ 35: 1293-1298, 2014. DOI: 10.3724/SP.J. 1008.2014.01293

10 Lee HM and Banini BA: Updates on chronic HBV: Current challenges and future goals. Curr Treat Options Gastroenterol 17: 271-291, 2019. PMID: 31077059. DOI: 10.1007/s11938019-00236-3

11 Hou J, Wang G, Wang F, Cheng J, Ren H, Zhuang H, Sun J, Li L, Li J, Meng Q, Zhao J, Duan Z, Jia J, Tang H, Sheng J, Peng J, Lu F, Xie Q and Wei L: Guideline of prevention and treatment for chronic hepatitis B (2015 Update). J Clin Transl Hepatol 5: 297318, 2017. PMID: 29226097. DOI: 10.14218/JCTH.2016.00019

12 Magdalena A. Budzinska, Nicholas A. Shackel, Urban S and Tu T: Cellular: Genomic sites of hepatitis B virus DNA integration. Genes 9: 365-370, 2018. PMID: 30037029. DOI: 10.3390/genes9070365

13 Lupberger J and Hildt E: Hepatitis B virus-induced oncogenesis. World J Gastroenterol 13: 74-81, 2007. PMID: 17206756. DOI: 10.3748/wjg.v13.i1.74

14 Martin-Lluesma S, Schaeffer C, Robert EI, van Breugel PC, Leupin $\mathrm{O}$, Hantz $\mathrm{O}$ and Strubin M: Hepatitis B virus $\mathrm{X}$ protein affects $\mathrm{S}$ phase progression leading to chromosome segregation defects by binding to damaged DNA binding protein 1 . Hepatol 48: 1467-1476, 2008. PMID: 18781669. DOI: 10.1002/hep.22542

15 Pollicino T, Saitta C and Raimondo G: Hepatocellular carcinoma: The point of view of the hepatitis B virus. Carcinogenesis 32: 1122-1132, 2011. PMID: 21665892. DOI: $10.1093 /$ carcin/bgr 108

16 Liang HW, Wang N, Wang Y, Wang F, Fu Z, Yan X, Zhu H, Diao W, Ding Y, Chen X, Zhang CY and Zen K: Hepatitis B virushuman chimeric transcript HBX-LINE1 promotes hepatic injury via sequestering cellular microRNA-122. J Hepatol 64: 278-291, 2016. PMID: 26409216. DOI: 10.1016/j.jhep.2015.09.013

17 Li X, Zhang J, Yang Z, Kang J, Jiang S, Zhang T, Chen T, Li M, Lv Q, Chen X, McCrae MA, Zhuang H and Lu F: The function of targeted host genes determines the oncogenicity of HBV integration in hepatocellular carcinoma. J Hepatol 60: 975-984, 2014. PMID: 24362074. DOI: 10.1016/j.jhep.2013.12.014

18 Lee WY, Bachtiar M, Choo CCS and Lee CG: Comprehensive review of hepatitis B virus-associated hepatocellular carcinoma research through text mining and big data analytics. Biol Rev 94: 353-367, 2019. PMID: 30105774. DOI: 10.1111/brv.12457

19 Hu B, Wang R, Fu J, Su M, Du M, Liu Y, Li H, Wang H, Lu F and Jiang $\mathrm{J}$ : Integration of hepatitis $\mathrm{B}$ virus $\mathrm{S}$ gene impacts on hepatitis B surface antigen levels in patients with antiviral therapy. J Gastroenterol Hepatol 33: 1389-1396, 2018. PMID: 29266382. DOI: $10.1111 /$ jgh.14075

20 Liu F, Wang X-W, Chen L, Hu P, Ren H and Hu H-D: Systematic review with meta-analysis: Development of hepatocellular carcinoma in chronic hepatitis B patients with hepatitis B surface antigen seroclearance. Aliment Pharmacol Ther 43: 1253-1261, 2016. PMID: 27117732. DOI: 10.1111/apt.13634

21 Li YW, Yang FC, Lu HQ and Zhang J-S: Hepatocellular carcinoma and hepatitis B surface protein. World J Gastroenterol 22: 19431952, 2016. PMID: 26877602. DOI: 10.3748/wjg.v22.i6.1943
22 Yang Y, Gao J, Tan YT, Li HL, Wang J, Ma X, Zheng W, Shu $\mathrm{XO}$ and Xiang YB: Individual and combined effects of hepatitis B surface antigen level and viral load on liver cancer risk. J Gastroenterol Hepatol 33: 1131-1137, 2018. PMID: 29065440. DOI: $10.1111 /$ jgh.14032

23 Zhang F, Wang Q, Ye L, Feng Y and Zhang X: Hepatitis B virus $\mathrm{X}$ protein upregulates expression of calpain small subunit 1 via nuclear factor- $\chi \mathrm{B} / \mathrm{p} 65$ in hepatoma cells. J Med Virol 82: 920928, 2010. PMID: 20419804. DOI: 10.1002/jmv.21753

24 Mignon AK: Morgan DO: The cell cycle: Principles of control, primers in biology. Cell Division 2: 27-35, 2007. DOI: 10.1186/1747-1028-2-27

25 Bok J, Kim KJ, Park MH, Cho SH, Lee HJ, Lee EJ, Park C and Lee JY: Identification and extensive analysis of invertedduplicated HBV integration in a human hepatocellular carcinoma cell line. BMB Rep 45: 365-370, 2012. PMID: 22732223. DOI: 10.5483/bmbrep.2012.45.6.279

26 Guo Y, Kang W, Lei X, Li Y, Xiang A, Liu Y, Zhao J, Zhang J and Yan Z: Hepatitis B viral core protein disrupts human host gene expression by binding to promoter regions. BMC Genomics 13: 563-570, 2012. PMID: 23088787. DOI: 10.1186/1471-2164-13-563

27 Haaß W, Kleiner H, Müller MC, Hofmann WK, Fabarius A and Seifarth W: Measurement of separase proteolytic activity in single living cells by a fluorogenic flow cytometry assay. PLoS One 10: e0133769, 2015. PMID: 26267133. DOI: 10.1371/ journal.pone. 0133769

28 Mukherjee M, Byrd T, Brawley VS, Bielamowicz K, Li XN, Merchant F, Maitra S, Sumazin P, Fuller G, Kew Y, Sun D,Powell SZ, Ahmed N, Zhang N and Pati D: Overexpression and constitutive nuclear localization of cohesin protease Separase protein correlates with high incidence of relapse and reduced overall survival in glioblastoma multiforme. J Neurooncol 119: 27-35, 2014. PMID: 24792645. DOI: 10.1007/ s11060-014-1458-6

29 Finetti P, Guille A, Adelaide J, Birnbaum D, Chaffanet M and Bertucci F: ESPL1 is a candidate oncogene of luminal B breast cancers. Breast Cancer Res Treat 147: 51-59, 2014. PMID: 25086634. DOI: 10.1007/s10549-014-3070-z

30 Mukherjee M, Ge G, Zhang N, Huang E, Nakamura LV, Minor M, Fofanov V, Rao PH, Herron A and Pati D: Separase loss of function cooperates with the loss of p53 in the initiation and progression of $\mathrm{T}$ - and B-cell lymphoma, leukemia and aneuploidy in mice. PLoS One 6: e22167, 2011. PMID: 21799785. DOI: 10.1371/journal.pone.0022167

31 Rao ZL, Dong J, Zhu YY, Chen J, You J, Zheng Q and Jiang JJ: Hepatitis B surface antigen affects the expression of lipid metabolism-related genes in HepG2 cells. Chinese J Hepatol 21: 624-630, 2013. PMID: 24119745. DOI: 10.3760/cma.j.issn.10073418.2013.08.014 